\title{
Order-parameter-based Monte Carlo simulation of crystallization
}

\author{
Manan Chopra \\ Department of Chemical Engineering, University of Wisconsin, Madison, Wisconsin 53706-1691 \\ Marcus Müller \\ Institut für Theoretische Physik, Georg-August Universität, 37077 Göttingen, Germany \\ J. J. de Pablo ${ }^{\text {a) }}$ \\ Department of Chemical Engineering, University of Wisconsin, Madison, Wisconsin 53706-1691
}

(Received 28 September 2005; accepted 27 January 2006; published online 4 April 2006)

\begin{abstract}
A Monte Carlo simulation method is presented for simulation of phase transitions, with emphasis on the study of crystallization. The method relies on a random walk in order parameter $\Phi\left(q^{N}\right)$ space to calculate a free energy profile between the two coexisting phases. The energy and volume data generated over the course of the simulation are subsequently reweighed to identify the precise conditions for phase coexistence. The usefulness of the method is demonstrated in the context of crystallization of a purely repulsive Lennard-Jones system. A systematic analysis of precritical and critical nuclei as a function of supercooling reveals a gradual change from a bcc to a fcc structure inside the crystalline nucleus as it grows at large degrees of supercooling. The method is generally applicable and is expected to find applications in systems for which two or more coexisting phases can be distinguished through one or more order parameters. (C) 2006 American Institute of Physics. [DOI: $10.1063 / 1.2178324]$
\end{abstract}

\section{INTRODUCTION}

Molecular simulations are used extensively to study phase transitions. Coexisting phases are often separated by large free energy barriers; conventional simulation techniques such as canonical molecular dynamics (MD) or Monte Carlo ${ }^{1}$ (MC) are unable to sample both phases simultaneously, and generally remain trapped in one or the other local free energy minimum. Several simulation techniques have been developed to overcome large free energy barriers: examples include umbrella sampling ${ }^{2}$ and its variants, ${ }^{3}$ multicanonical sampling, ${ }^{4}$ and parallel tempering formalisms. ${ }^{5}$ The basic idea behind umbrella or multicanonical sampling is to introduce a bias that favors configurations having a higher free energy at the expense of configurations with a lower free energy, thereby sampling thermodynamic space in a somewhat uniform manner. Umbrella sampling requires that a good initial guess of the biasing potential be provided, followed by an iterative optimization process. Multicanonical sampling requires calculation of a biasing function $w\left(\Phi\left(q^{N}\right)\right)$, where $q^{N}$ is a set of coordinates and $\Phi$ is an order parameter, which must also be obtained through an iterative procedure. Recently, Wang and Landau ${ }^{6}$ have proposed a remarkably simple and effective algorithm (WL algorithm) for uniform sampling of energy space. In WL sampling, a random walk is performed in energy space, and configurations having different energies are visited with the same frequency. Since its original conception, Wang-Landau sampling has been extended to simulations in a continuum and to different ensembles. $^{7-11}$ Several improvements have also been proposed to extend its range of applicability; these include the

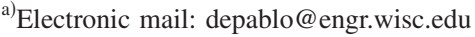

use of configurational temperature to improve the convergence of the algorithm, ${ }^{12}$ its combination with parallel tempering or replica exchange Monte Carlo techniques, ${ }^{12,13}$ $N$-fold way Monte Carlo, ${ }^{14}$ and optimization of round trip times $^{15}$ to improve the accuracy of the method. Note that such implementations, however, implicitly assume that a random walk in energy space leads to a random walk in configuration space. There are numerous cases of interest where this is not the case; these cases include the problem of crystallization.

Based on Wang and Landau's ideas, we have introduced a series of simulation techniques, which we collectively refer to as the expanded ensemble density of states (EXEDOS) sampling, that permits systematic calculation of free energy profiles in terms of arbitrary reaction coordinates or order parameters. $^{16-18}$ Various implementations of the EXEDOS sampling have been used to examine the free energy of colloidal particles suspended in liquid crystals as a function of their separation, ${ }^{17}$ or the free energy required to fold and unfold a protein as a function of its end-to-end distance. ${ }^{16}$ In those implementations of EXEDOS sampling, $w\left(q^{N}\right)$ need not be known a priori; it is determined "on the fly" in a self-adjusting manner. The EXEDOS approach prescribes that a random walk be performed in an expanded ensemble expressed in terms of one or several reaction coordinates or order parameters. In this work, a method is presented in which the weights obtained from an EXEDOS simulation are related to the free energy as a function of the order parameter (Landau free energy). ${ }^{19}$ This technique permits precise calculation of the free energy profile of an arbitrary system as it undergoes a phase transition. The free energy corresponds to the equilibrium free energy of the macrostates characterized by a fixed value of the order parameter. The path along 
which the transition occurs depends on the specific choice of the order parameter, and it need not coincide with the states visited during the kinetics of phase transformation-a nonequilibrium process. The use of the method is illustrated in the context of crystallization, which continues to pose considerable challenges to numerical simulation. ${ }^{20-23}$

\section{EXPANDED ENSEMBLE DOS METHOD}

Consider an EXEDOS simulation of a system consisting of $N$ particles, at temperature $T$ and pressure $P$; configurations are sampled with probability ${ }^{16,17}$

$$
\mathcal{P}_{w, \beta, P}\left(q^{N}\right)=\frac{\exp \left[-\beta U\left(q^{N}\right)-\beta P V\left(q^{N}\right)\right] w\left(\Phi\left(q^{N}\right)\right)}{Q_{w}(N, P, T)},
$$

where $w\left(\Phi\left(q^{N}\right)\right)$ is the weight associated with an order parameter $\Phi\left(q^{N}\right)$, and $Q_{w}(N, P, T)$ is the normalization constant. Subscript $w$ denotes a sampling corresponding to a weighted ensemble.

In EXEDOS, a running average of the weights can be computed and refined in a self-adjusting manner. Ideally, the weights are related to the unweighed ensemble distribution by

$$
w\left(\Phi\left(q^{N}\right)\right)=\frac{1}{\mathcal{P}_{\beta, P}\left(\Phi\left(q^{N}\right)\right)},
$$

where $\mathcal{P}_{\beta, P}\left(\Phi\left(q^{N}\right)\right)$ is, in the present study of crystallization, the isobaric-isothermal $(N, P, T)$ ensemble probability distribution of $\Phi\left(q^{N}\right)$.

The method presented here requires that an appropriate order parameter be identified. The order parameter must clearly distinguish any coexisting phases from each other. Examples of suitable order parameters include the scalar order parameter for the study of nematic-isotropic transitions in liquid crystals, ${ }^{24}$ a density based order parameter $\psi$ for block copolymer systems,${ }^{25}$ or a bond order parameter for the study of crystallization. ${ }^{26}$ Having specified a suitable order parameter, EXEDOS simulations are performed to calculate $\mathcal{P}_{\beta, P}\left(\Phi\left(q^{N}\right)\right)$. The Landau free energy of the system $\Lambda(\Phi)$ can then be related to $\mathcal{P}_{\beta, P}\left(\Phi\left(q^{N}\right)\right)$ by ${ }^{19}$

$$
\Lambda\left(\Phi\left(q^{N}\right)\right)=\text { const }-k_{B} T \ln \left\{\mathcal{P}\left(\Phi\left(q^{N}\right)\right)\right\} .
$$

Depending on whether $\mathcal{P}(\Phi)$ is obtained from EXEDOS in a constant $(N, V, T)$ or constant $(N, P, T)$ ensemble, $\Lambda$ will correspond to the Helmholtz free energy or the Gibbs free energy of the system, respectively.

Away from a phase transition, $\Lambda$ is expected to exhibit a single minimum as a function of $\Phi$. Near coexistence, however, $\Lambda$ develops two minima; the barrier between the minima corresponds to the free energy associated with the formation of an interface. ${ }^{27}$ The method described above is capable of providing a free energy profile (including free energy barriers) at any given temperature and pressure. In general, however, the coexistence temperature (e.g., melting temperature) or pressure is not known. In order to obtain the precise conditions under which two phases coexist it is therefore necessary to conduct a series of exploratory simulations, until a set of conditions is found for which the free energy minima corresponding to the two equilibrium states become identical. This process can be facilitated by resorting to the following histogram extrapolation scheme. ${ }^{28}$ Energy and volume data from EXEDOS simulations must be saved and sorted in joint histograms according to the order parameter. The probability of a system to be in bin $m$, denoted by $\mathcal{P}_{\beta_{2}, P}\left(\Phi_{m}, \Phi_{m+1}\right)$, at a temperature $\beta_{2}$ can be obtained from a simulation at a temperature $\beta_{1}$ from

$$
\mathcal{P}_{\beta_{2}, P}\left(\phi_{m}, \phi_{m+1}\right)=\frac{\mathcal{P}_{\beta_{1}, P}\left(\phi_{m}, \phi_{m+1}\right) \sum_{i=1}^{N_{m}} \exp \left(\left(\beta_{1}-\beta_{2}\right)\left(U_{i, m}+P V_{i, m}\right)\right)}{\sum_{m=1}^{N_{b}} \mathcal{P}_{\beta_{1}, P}\left(\phi_{m}, \phi_{m+1}\right) \sum_{i=1}^{N_{m}} \exp \left(\left(\beta_{1}-\beta_{2}\right)\left(U_{i, m}+P V_{i, m}\right)\right)},
$$

where $U_{m, i}$ and $V_{m, i}$ are the energy and volume entries in the $m$ th order parameter histogram bin, $N_{b}$ is the total number of histogram bins, and $N_{m}$ is the number of entries in bin $m$. The collection of thermodynamic data is done only after the histograms have become sufficiently flat. Equation (4) can be used to determine the precise values of coexistence temperature $T^{*}$ and pressure $P^{*}$ for which the free energy Eq. (3) exhibits two minima of equal weight. ${ }^{29}$ This histogram extrapolation scheme works particularly well in conjunction with EXEDOS because we do not only sample configurations having large Boltzmann weights but also configurations that have a small Boltzmann weight but that are important for free energy estimations. Configurations having a small weight away from coexistence could be relevant at coexistence; the sampling of such configurations in EXEDOS simu- lations therefore facilitates histogram extrapolation.

Once the free energy profile of a system is obtained and it is verified that the system adopts a slablike configuration with two interfaces [cf. Fig. 6(b)] at the free energy barrier, the excess free energy $\Delta \Lambda$ can be related to the interfacial tension by $^{27}$

$$
\gamma=\frac{\Delta \Lambda}{2 L^{2}},
$$

where $L$ is the dimension of the box. The factor of 2 in the denominator arises because of the presence of two interfaces in a simulation cell with periodic boundary conditions. Equation (5) is only accurate for large systems with minimal finite-size effects. Alternatively, the interfacial tension can be obtained by using the finite-size scaling approach of 
Binder. $^{27}$ In that case, the interfacial tension, $\gamma_{L}$, calculated from a finite system of size $L$ can be related to the true, infinite-system size interfacial tension $\gamma$ through

$$
\beta \gamma_{L} \equiv \frac{\beta \Delta \Lambda_{L}}{2 L^{2}}=\frac{a+b \ln L}{L^{2}}+\beta \gamma,
$$

where $a$ and $b$ are system-specific constants.

Having determined the free energy $\Lambda$ as a function of $\Phi\left(q^{N}\right)$, configurations corresponding to the top of the free energy barrier can be used to explore the transition pathways from one phase to another, as will be discussed later in this work in the context of nucleation mechanisms. ${ }^{21}$

\section{APPLICATION TO A LENNARD-JONES CRYSTAL}

To demonstrate the usefulness of the technique outlined above, we have chosen to simulate the crystallization of a model about which much is known from previous simulations, namely, a system of particles interacting through a repulsive Lennard-Jones (LJ) potential $U(r)=\epsilon(\sigma / r)^{12}$ truncated at a distance of $r_{c}=2 \sigma$. The long-range contribution to the potential was calculated under the assumption of constant density. For this system, the stable nuclei at melting are believed to be face centered cubic (fcc).$^{30-32}$ Previous simulations by ten Wolde et al. ${ }^{21}$ used umbrella sampling to generate free energy curves and to examine the structure of the critical nuclei that form in a deeply supercooled liquid.

The order parameter used is the bond orientational order parameter, $Q_{6}$, originally introduced by Steinhardt et al. ${ }^{26}$ and later used by van Duijneveldt and Frenkel ${ }^{20}$ to study crystallization. It is sensitive to the overall degree of crystallinity in the system, irrespective of the crystal structure, i.e., it distinguishes the liquid from the crystal. The values of $Q_{6}$ for pure fcc, body centered cubic (bcc), and liquid are $0.57452,0.51069$, and 0.0, respectively. Previous work ${ }^{20}$ has shown that a defective fcc crystal, which crystallizes from the liquid in simulations, has an order parameter below 0.5 , and that finite-size effects lead to small positive values for the order parameter in the liquid phase.

Our simulations were performed on systems of various sizes, ranging from 108 to 1364 particles. The order parameter range explored here goes from 0.05 to 0.5 for the small system and from 0.02 to 0.45 for the larger systems. In all simulations the order parameter was calculated at every step and was used in the Monte Carlo acceptance criteria. Following our previous work, the range of order parameter was split into multiple overlapping windows (intervals of order parameter). ${ }^{16,17}$ Ten overlapping windows with a $50 \%$ of overlap between adjacent windows were employed in all simulations. Configuration swaps were implemented to facilitate our calculations. During the simulation we observed the tunneling of individual replicas between the liquid and the crystalline state. The total number of tunneling events for each system size exceeds $\#=20000$. Thus the statistical uncertainty of the free energies we calculate is on the order of $\Delta \Lambda \sim O\left(k_{B} T / \sqrt{\#}\right) \approx 0.01 k_{B} T$. The corresponding error $\Delta T^{*}$ (in reduced LJ units) is of the order $\approx 0.001$ and $\Delta P^{*}$ is of the order $\approx 0.003$. Figure 1 shows a small part of the history of a replica traveling along the order parameter coordinate.

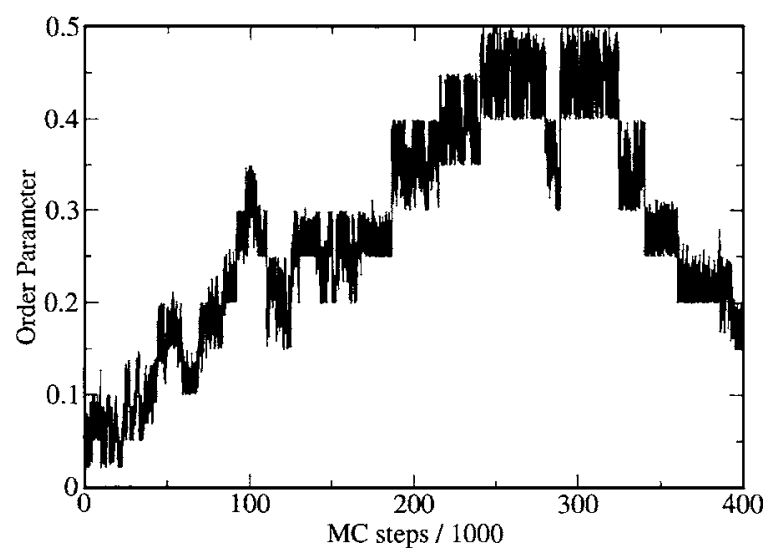

FIG. 1. A small range of Monte Carlo steps showing change of order parameter for a single replica in EXEDOS simulation.

Figure 1 shows how one out of the ten replicas moves from low values of the order parameter to large values, before coming back to low values. The complete order parameter trajectory is analogous to the small fragment shown in Fig. 1.

The accuracy of the simulated coexistence temperature and pressure in the method outlined in this work appears to be comparable (or sometimes better) to that achieved with techniques such as phase switch Monte Carlo, ${ }^{33}$ selfreferential Monte Carlo, ${ }^{34}$ and techniques that rely on thermodynamic integration and Gibbs-Duhem integration. ${ }^{35}$ The added advantage of the order-parameter-based method is that, in addition to obtaining accurate coexistence conditions, we also get an accurate free energy curve as a function of the chosen reaction coordinate. We note here that an alternative technique, the so-called transition path sampling Monte Carlo, can also lead to accurate coexistence conditions and a free energy curve. In a recent study of crystallization, ${ }^{36}$ the free energy barrier and a transition path were presented for the Lennard-Jones fluid. One disadvantage of transition path sampling, however, is that it is much more computationally demanding than the technique proposed here.

The EXEDOS simulations were initially performed on the smaller system at a reduced LJ temperature $\left(T^{*}\right.$ $\left.\equiv k_{B} T / \epsilon\right) \quad T^{*}=1.0 \quad$ and pressure $\left(P^{*} \equiv P \sigma^{3} / \epsilon\right) \quad P^{*}=24.21$, which are the estimates for the coexistence conditions in the literature $^{20}$ for a system of similar size. Figure 2 shows the resulting free energy curve. It can be seen that, for our system geometry, $T^{*}=1.0$ is not the best finite-size estimate of the coexistence temperature. Using Eq. (4), we reweight the free energy histograms to identify the temperature where the two minima become identical. This condition is fulfilled at $T_{\sigma}^{*}=1.14$. A second set of EXEDOS simulations were performed at this new temperature to explore the range over which data can be reweighed with the single-histogram extrapolation of Eq. (4). The results of that second simulation are also shown in Fig. 2, and are consistent with our extrapolated results of the previous run in several respects: (i) The barrier height in both curves is about ten LJ units $\left(E^{*}\right.$ $=E / \epsilon$ ), (ii) the free energy minimum for the crystalline phase shifts to the left when temperature increases, and (iii) the free energy minima are almost identical in both curves.

For a larger system, 864 particles, our starting guess for 


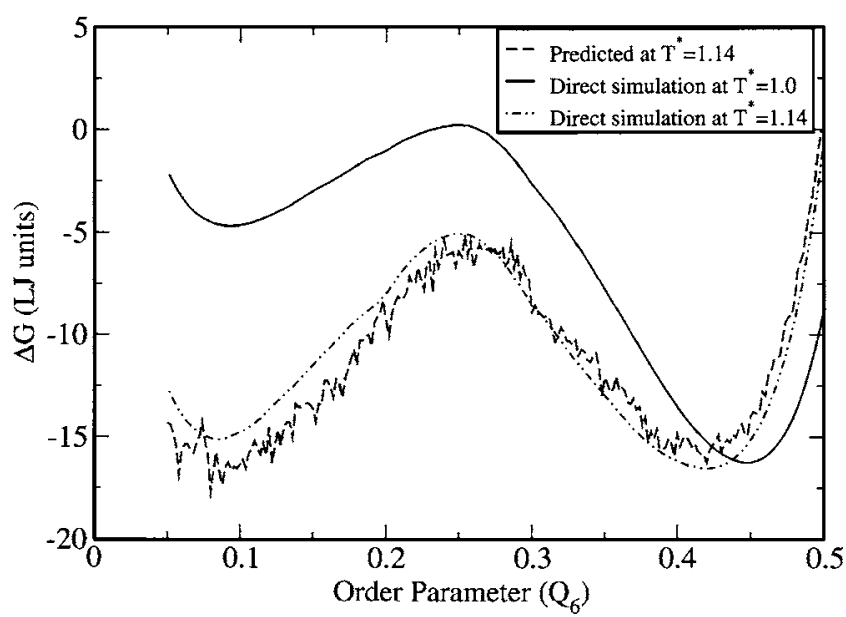

FIG. 2. Free energy profile of a repulsive LJ system containing 108 particles: EXEDOS simulations at $T^{*}=1.0$ (solid line), predicted free energy profile by reweighing $T^{*}=1.0$ data to $T^{*}=1.14$ (dash line), and EXEDOS simulations at $T^{*}=1.14$ (dot-dot-dash line).

coexistence temperature and pressure was $T^{*}=1.14$ and $P^{*}$ $=24.21$. The order parameter range was set between 0.02 and 0.45 . EXEDOS simulations were again performed in multiple windows. The free energy curves obtained at this temperature and pressure (see Fig. 3) show that, as a result of finite-size effects, $T^{*}=1.14$ is not the estimate of the phase transition temperature for the larger system. The free energy curves were therefore reweighed to arrive at $T_{\sigma}^{*}=1.03$ as an estimate for the melting temperature. The histogramextrapolated free energy profile was again confirmed by a subsequent EXEDOS simulation at the same temperature. The above results are indicative of strong finite-size effects; these effects are larger than the statistical errors associated with our simulations and limit the accuracy of our estimated melting temperatures. It is therefore important to use finitesize scaling to predict the coexistence temperature for an infinite system. Figure 4 shows the variation of $T_{\sigma}^{*}$ with the volumetric length scale. The figure includes results for systems of 256 and 500 particles; a clear finite-size scaling can be seen in the behavior of $T_{\sigma}^{*}$ with volume. The extrapolation

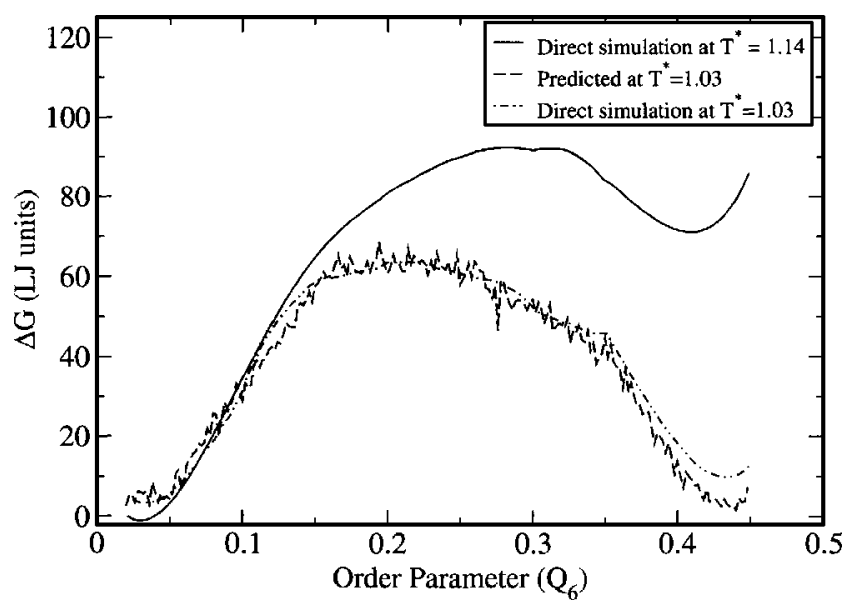

FIG. 3. Free energy profile of a repulsive LJ system containing 864 particles: EXEDOS simulations at $T^{*}=1.14$ (solid line), predicted free energy profile by reweighing $T^{*}=1.14$ data to $T^{*}=1.03$ (dash line), and EXEDOS simulations at $T^{*}=1.03$ (dot-dot-dash line). to infinite size yields a coexistence temperature of $T_{\sigma, \infty}^{*}$ $=0.99(1)$. Note that in the algorithm proposed here the nucleation barrier necessary to expand a solid nucleus is removed, but the barrier associated with transforming an ordered but homogeneous liquid into an inhomogeneous liquid with an initial solid nucleus at the same value of the order parameter, $Q_{6}$, remains. ${ }^{37,38}$ This formation of solid bodies of high $Q_{6}$ allows the larger system to move more easily between high and low order parameters, thereby facilitating the convergence of the simulations.

A subsequent structural examination of the trajectories was performed using Voronoi tessellation and employing the analysis outlined by ten Wolde $e t$ al. ${ }^{21}$ In this procedure, a combined distribution of local invariants $q_{4}, q_{6}, w_{4}$, and $w_{6}$ was generated for a given configuration. A detailed explanation of local invariants $q_{4}, q_{6}, w_{4}$, and $w_{6}$ can be found in the original reference. ${ }^{44}$ The distribution of local invariants was then decomposed into distributions for thermally equilibrated bcc, fcc, and liquid configurations (see Fig. 5) according to

$$
\Delta^{2}=\left[\widetilde{\nu}_{\mathrm{cl}}-\left(f_{\text {liq }} \widetilde{\nu}_{\text {liq }}+f_{\text {bcc }} \widetilde{\nu}_{\mathrm{bcc}}+f_{\text {fcc }} \widetilde{\nu}_{\text {fcc }}\right)\right]^{2},
$$

where $\widetilde{\nu}_{\mathrm{cl}}, \widetilde{\nu}_{\text {liq }}, \widetilde{\nu}_{\mathrm{fcc}}$, and $\widetilde{\nu}_{\mathrm{bcc}}$ are combined distributions for the cluster to be analyzed, thermally equilibrated liquid, fcc, and bcc configurations, respectively. In Eq. (7) $f_{\text {liq }}, f_{\text {bcc }}$, and $f_{\text {fcc }}$ are weight coefficients for liquid, bcc, and fcc configurations. The analysis confirmed that the first minimum in the free energy profile corresponds to a liquid phase, and the second minimum corresponds to a thermally equilibrated fcc phase.

The analysis also provides an explanation for the asymmetric shape and the kinks that appear in the free energy curve of Fig. 3. These features were not observed in previous simulations of the same system, which used a different simulation approach, and it is therefore important to discuss their origin by investigating the configurations that the system adopts as a function of the order parameter. ${ }^{37-42}$ Between the order parameter of 0.10 and 0.15 , simulated configurations reveal the occurrence of spherical nuclei. In the order parameter range from 0.15 to 0.25 we observe the formation of solid-fluid interfaces. An interesting point to note here is that the interface has a predominantly bcc character, hinting at the formation of thin interfacial layers which are structurally different from the bulk solid. The fact that we observe a bcc interface coincides with the fact that the liquid-bcc tension is smaller than the liquid-fcc tension. ${ }^{22}$ Moving further along the free energy profile to the range of $0.25-0.35$ we see the growth of an interface that spans the entire simulation box, with a defective bcc-like solid. Upon further increasing the order parameter we observe that, beyond 0.35 , this defective bcc-like solid rearranges into a defective fcc-like solid. Figure 6 shows snapshots of various representative configurations extracted from our simulations. The transitions to and from various configurations in EXEDOS are highly reversible. The asymmetric shape of the free energy profile can be attributed to this two-step transition from an interface between a liquid and defective bcc-like structures, and then from defective bcc-like structures to defective fcc-like structures. 


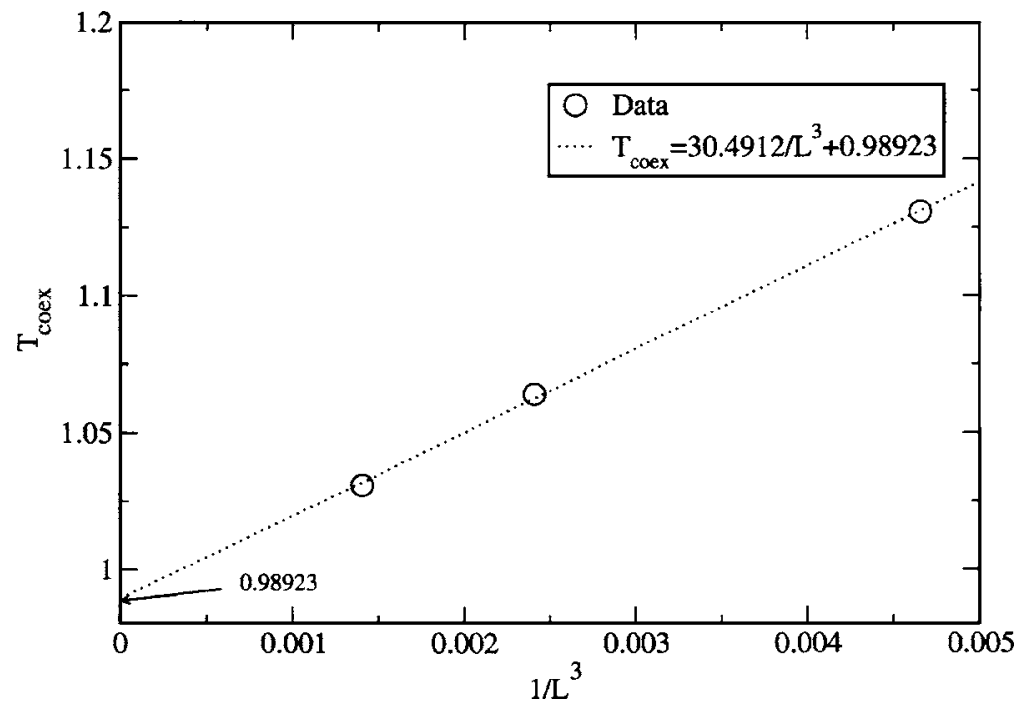

FIG. 4. $T_{\sigma}^{*}$ as a function of system size; three different system sizes of 256,500 , and 864 particles was used to see the finite-size scaling. The arrow points to the limiting value.

The fact that solid-liquid interfaces can form in our simulations can be exploited to calculate the interfacial tension of the system. To this end, simulations were performed for four system sizes, namely, 108, 256, 500, and 864 particles. From the simulations $\left(T^{*}=1.03\right)$ of the above systems, free energy barriers were calculated as shown in Fig. 8. The interfacial tension from this analysis is 0.31 in LJ units, with an error of magnitude of $0.01 \mathrm{LJ}$ unit (same as that for the free energy calculations). Previous studies ${ }^{22,23}$ for repulsive LJ systems interacting with an $r^{-n}$ potential $(n=8)$ have reported $\gamma \approx 0.5$ (for $T^{*}=1.03$ ) for a bcc solid in contact with a liquid phase. This value is expected to decrease for $n=12,{ }^{23}$ and therefore the interfacial tension calculated in this work appears to be compatible with literature results. Figure 7 shows the results of this finite-size analysis. Two remarks are in order: (i) We note that the finite-size effects are surprisingly large. (ii) Since it is difficult to distinguish between the two correction terms in Eq. (6), our estimates for $\gamma$ depend strongly on the range of system sizes employed for subsequent extrapolations. As mentioned above, the solid phase in our interfacial configurations exhibits a bcc-like character; the interfacial tension calculated above is therefore that between a bulk bcc and a liquid phase, and not between a fcc

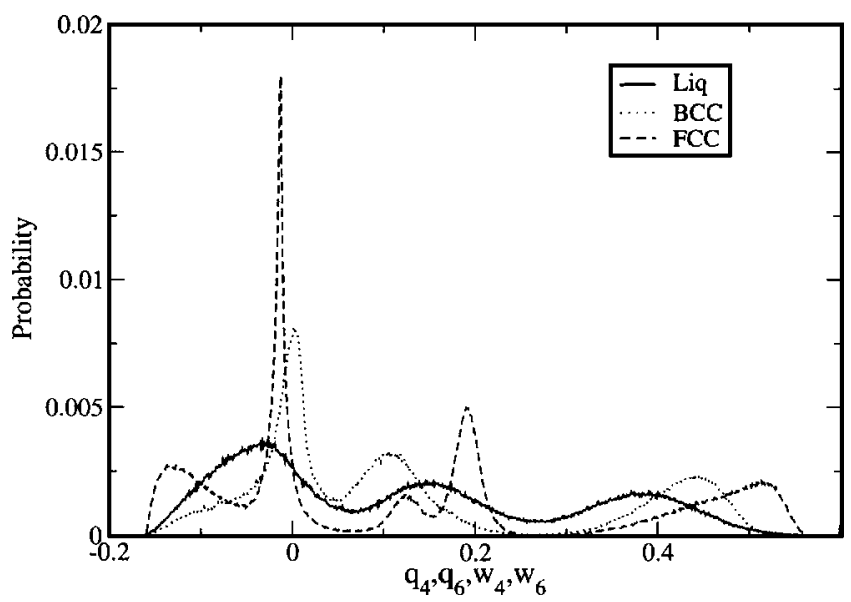

FIG. 5. The normalized probability histograms of different local invariants for thermally equilibrated liquid, bcc, and fcc configurations. solid and a liquid phase. For much larger system sizes we expect the interfacial configurations to consist of slabs of the two thermodynamically stable, coexisting phases-liquid and fcc solid-separated by two interfaces. Our results imply that there is a bcc layer of finite thickness at these interfaces. In view of the more complicated internal structure of the interfaces we also expect additional contributions to Eq. (6) that could arise, e.g., from the long-range elastic interactions between the two interfaces across the crystalline portion (which could affect the finite-size dependence of the measured interfacial tension). Similar difficulties in extracting an accurate value of the interfacial tension from finite-size scaling have been encountered at interfaces between the isotropic and nematic phases of rods. ${ }^{43}$

It is instructive to compare the value of interfacial tension predicted by the finite-size extrapolation above with that estimated on the basis of classical nucleation theory (CNT). The radius $R$ of the solid nucleus can be estimated by

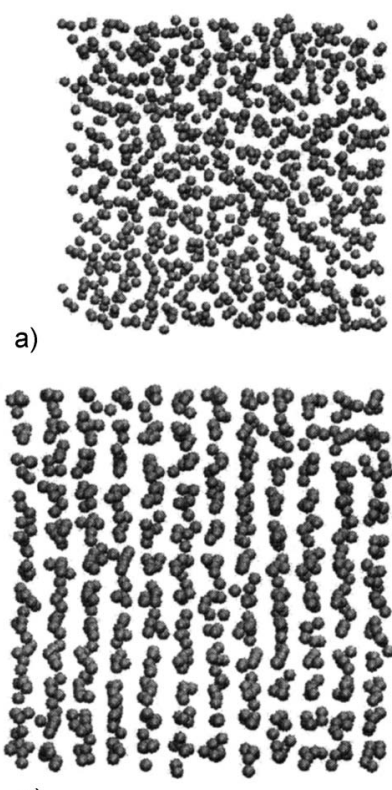

c)

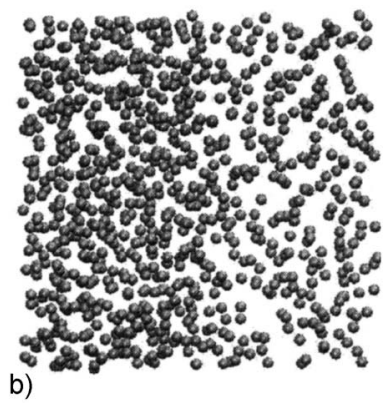

6.800 86480898 28300000 - $46 \% 408$ $68 \% 8 \% 0$ \%

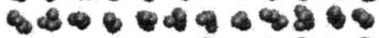
- $40.8 \%$ - 4848868 18088088 - $40 \% 8 \%$

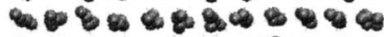

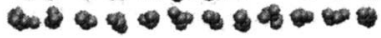

d)
FIG. 6. Snapshots of various configurations seen in a typical trajectory. 


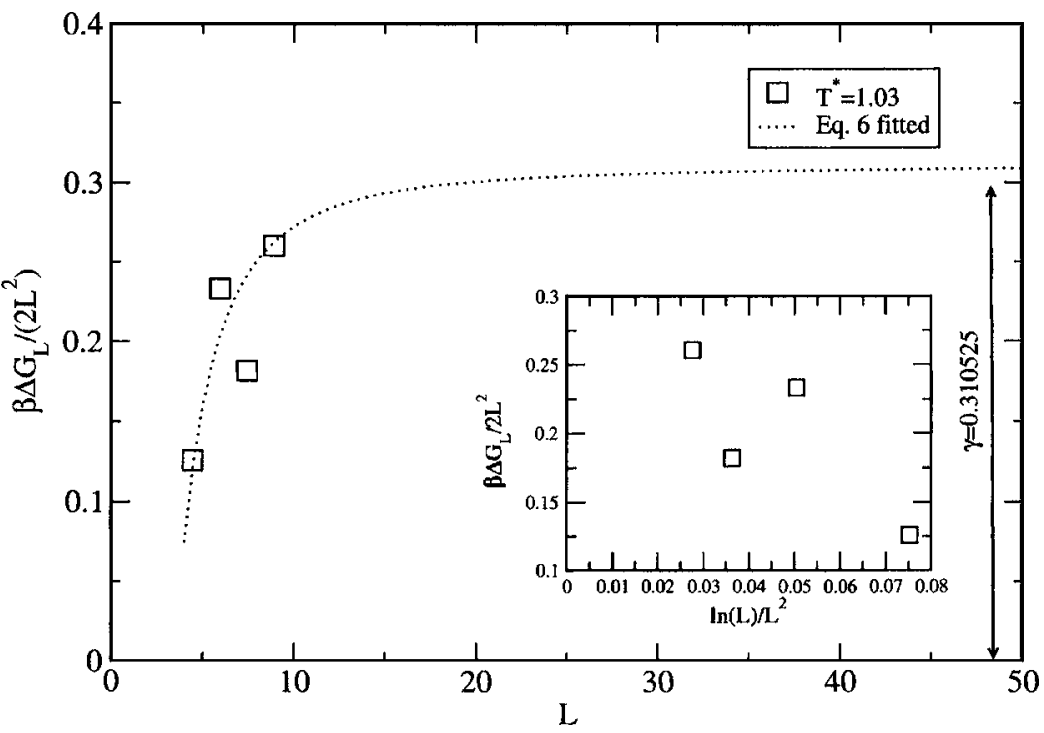

FIG. 7. Interfacial tension calculated from simulations of $108,256,500$, and 864 particle systems at $T^{*}=1.03$; lines show a fit of Eq. (6) to the data (dot-dot line).

$$
\frac{Q_{6}-Q_{6}^{L}}{(4 \pi / 3) R^{3}}=\frac{\left(Q_{6}^{C}-Q_{6}^{L}\right)}{V_{C}} .
$$

The above equation is a "lever rule" expression stating that the amount of solid in the system should be proportional to its $Q_{6}$ order parameter. In Eq. (8), $Q_{C}$ and $Q_{L}$ are order parameters for the crystal and the liquid phase, and $V_{C}$ is the volume for the crystal phase. The value of $Q_{C}$ is taken as that of a thermally equilibrated bcc crystal, 0.38 , and $Q_{L}$ is taken to be 0.04 . To find the radius $R$ from Eq. (8) we should use the value of $Q_{6}^{C}$ that corresponds to a defective bcc crystal. This value, $R$, can then be substituted in the free energy expression of CNT,

$$
\Delta G\left(Q_{6}\right)=4 \pi \gamma R^{2}-\frac{4 \pi}{3} R^{3} \frac{\Delta \mu}{v_{C}},
$$

where $\gamma$ denotes the interfacial tension between the liquid and the bcc structure inside the nucleus, and $\Delta \mu$ is the free energy difference between the liquid and the bcc crystal per particle. Since in our simulations we do not spontaneously form a stable bcc crystal—defective bcc crystals transform into fcc crystals - we have extrapolated the free energy curve corresponding to the defective bcc crystal to estimate $\Delta \mu$. The construction is shown in Fig. 8; we estimate the free energy difference between the liquid and the hypothetical bec crystal to be $35 k_{B} T$ for this system size. This value is similar to that employed in Ref. 20 namely, $40 k_{B} T$. Equation (9) can be used to fit our data in the $0.10-0.15$ region for the 864-particle system. The curve below 0.1 is shown to describe what CNT would predict. Figure 3 yields $\gamma=0.27$, which is in reasonable agreement with the estimate provided by finite-size scaling. Figure 8 shows the free energy curve predicted by CNT.

\section{EFFECT OF SUPERCOOLING}

Several literature reports have discussed the change in the structure of the critical nucleus as a function of changes in the bond order parameter. ${ }^{21,44}$ It is also of interest to consider how the structure of the critical nucleus changes with the extent of supercooling. An ensemble of configurations

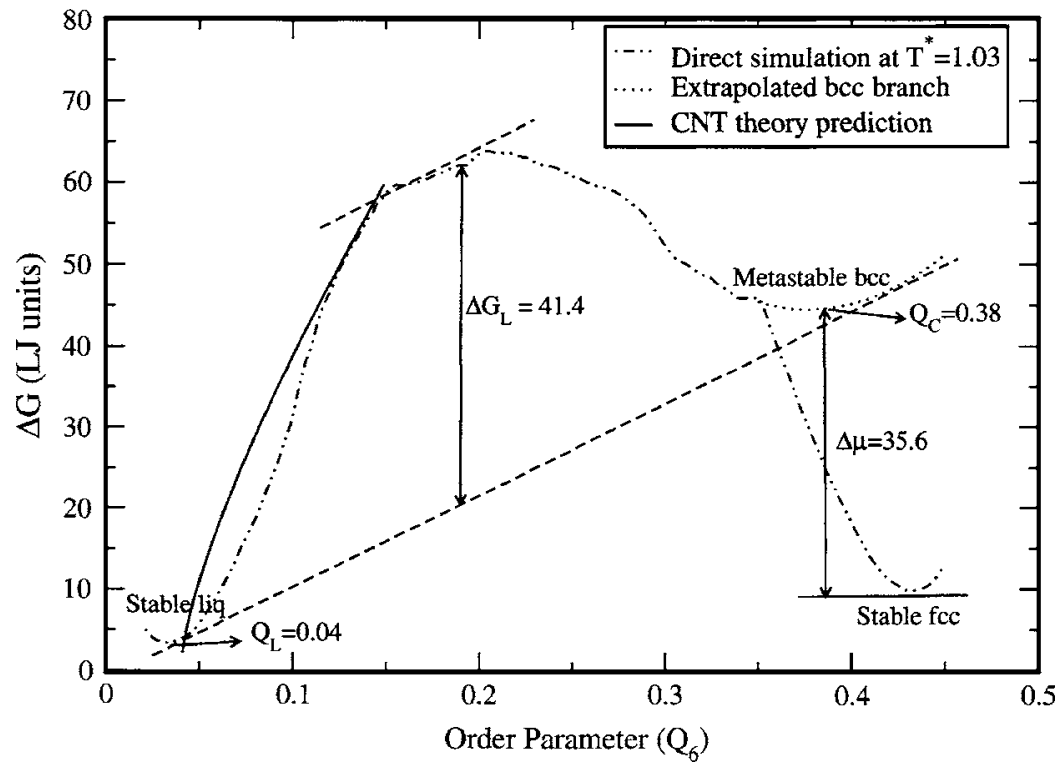

FIG. 8. Free energy profile predicted by CNT (solid line), free energy profile obtained from direct simulation (dot-dot-dash line), and extrapolated bcc branch (dot-dot line); $\Delta \mu=35.6$ and $\Delta G_{L}=41.4$. 


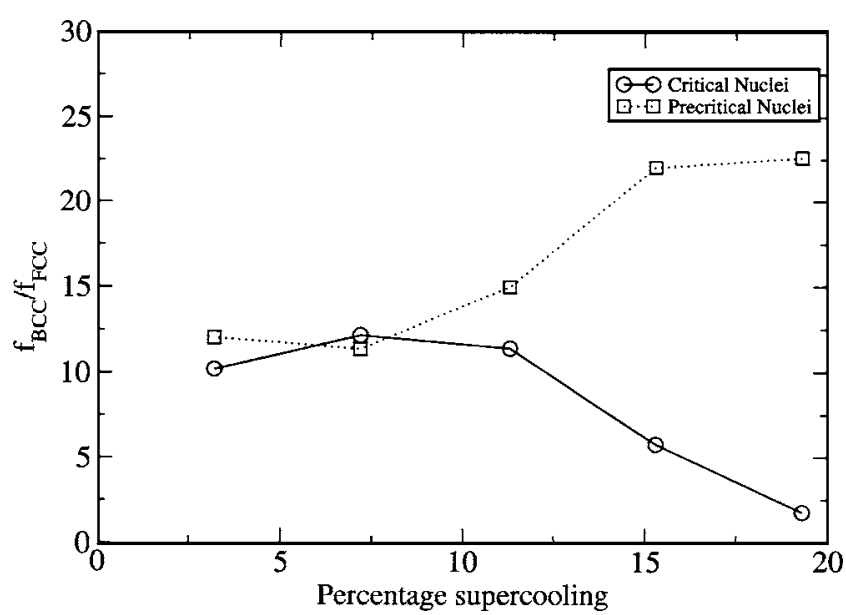

FIG. 9. Structural composition of critical and precritical nuclei at different degrees of supercooling.

corresponding to the top of the free energy curve was examined to extract the structural properties of the critical nucleus. In this work, nuclei formed at the top of the barrier are called critical, and nuclei formed just before the barrier are referred to as precritical. For example, in the case of 864 particles the nuclei formed in the order parameter range in the vicinity of the free energy peak ( 0.05 order parameter unit on both sides of the peak) are called critical, and nuclei formed just before the peak ( 0.05 below the critical nuclei range) are called precritical. The properties of critical and precritical nuclei are always calculated by averaging over all the configurations in the relevant order parameter range. Several literature reports have discussed the change in the structure of critical nuclei as a function of changes in the bond order parameter. Most of these studies have been conducted at constant supercooling, in the vicinity of $20 \%$. Note, however, that previous works relied on a coexistence temperature which differs significantly from that calculated here by finite-size scaling, and the actual extent of supercooling may have been different from the reported extent of supercooling. We consider how the structure of the critical nucleus changes with supercooling. By applying reweighing techniques, we can generate high-precision free energy curves for different degrees of supercooling, and then generate an ensemble of critical nuclei along the path characterized by the order parameter. Figure 9 shows the free energy profiles at different temperatures obtained by histogram reweighing.

The structural analysis of critical nuclei was again performed using the procedure outlined in the literature. ${ }^{21}$ As there were many precritical nuclei in the system, the properties of precritical nuclei were calculated by averaging over all the nuclei formed in the simulation. The size of precritical nuclei ranged from 25 to 50 particles. That of critical nuclei ranged from 100 to 200 particles. Figure 10 shows that, as we increase the degree of supercooling, the bcc character of the precritical nuclei increases. At the same time, the bcc character of the critical nuclei decreases. These results reveal an important aspect of crystallization that has generally been overlooked: at high degrees of supercooling, a substantial structural rearrangement takes place in going from a (small) precritical nucleus to a critical nucleus. However, this is not

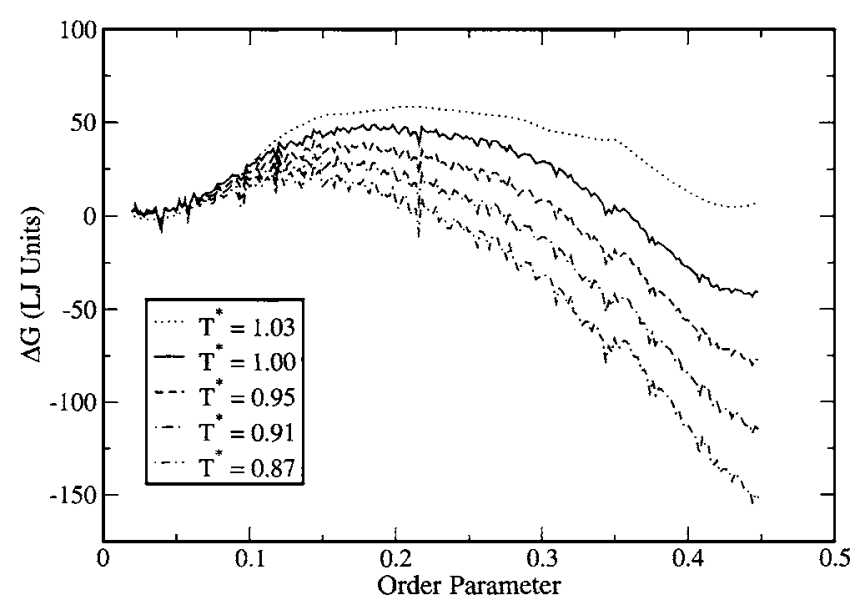

FIG. 10. Free energy profiles obtained at different temperatures using histogram reweighing.

the case for a low degree of supercooling, where the precritical nuclei can already be rather large. Unfortunately, the mechanism underlying such a rearrangement cannot be unambiguously deduced from our work. In the past, kinetic explanations have been advanced for the structural rearrangement of critical nuclei, ${ }^{44}$ but thermodynamic explanations of the structural changes, e.g., explanations based on the effects of local pressure, surface tension, and the shape of nuclei, are also conceivable.

\section{CONCLUSIONS}

In summary, we have presented a simple and effective simulation technique that permits calculation of the free energy profile associated with a phase transition, including that between a crystal and a liquid, with a high degree of precision. The method relies on density of states Monte Carlo sampling in an expanded ensemble defined in terms of one or more order parameters. It can be implemented within a parallel tempering framework, thereby facilitating its implementation on parallel processors. For crystallization, we use the bond orientation as an order parameter. The concomitant free energy curves are accurate and precise and, as shown in this work, can easily be reweighed to arrive at a relatively complete characterization of melting curves, extent of supercooling, and critical (or precritical) nuclei. An additional byproduct of the proposed method is that it permits calculation of the solid-liquid (or vapor-liquid) surface free energy, a quantity that is otherwise difficult to determine in simulations but that is essential for thermodynamic analysis of crystallization.

\section{ACKNOWLEDGMENTS}

The authors are grateful to Ethan Mastny for helpful discussions. This work is supported by the NSF-funded UWMRSEC on Nanostructured Interfaces.

\footnotetext{
${ }^{1}$ D. Frenkel and B. Smit, Understanding Molecular Simulations-From Algorithms to Applications (Academic, New York, 1996).

${ }^{2}$ G. M. Torrie and J. P. Valleau, Chem. Phys. Lett. 28, 578 (1974).

${ }^{3}$ P. Virnau and M. Müller, J. Chem. Phys. 120, 10925 (2004).

${ }^{4}$ B. Berg and T. Neuhaus, Phys. Lett. B 267, 249 (1991).

${ }^{5}$ C. J. Geyer and E. A. Thompsom, J. Am. Stat. Assoc. 90, 909 (1995).
} 
${ }^{6}$ F. Wang and D. P. Landau, Phys. Rev. Lett. 86, 2050 (2001).

${ }^{7}$ Q. Yan, R. Faller, and J. J. de Pablo, J. Chem. Phys. 116, 8745 (2002).

${ }^{8}$ R. Faller and J. J. de Pablo, J. Chem. Phys. 119, 4405 (2003).

${ }^{9}$ A. Ethan and J. J. de Pablo, J. Chem. Phys. 122, 124109 (2005).

${ }^{10}$ N. Rathore, T. A. Knotts IV, and J. J. de Pablo, J. Chem. Phys. 118, 4285 (2003).

${ }^{11}$ B. J. Schulz, K. Binder, and M. Müller, Phys. Rev. E 71, 046705 (2005).

${ }^{12}$ Q. Yan and J. J. de Pablo, Phys. Rev. Lett. 90, 035701 (2003).

${ }^{13}$ N. Rathore and J. J. de Pablo, J. Chem. Phys. 116, 7225 (2002).

${ }^{14}$ B. J. Schulz, K. Binder, and M. Müller, Int. J. Mod. Phys. C 13, 477 (2002).

${ }^{15}$ P. Dayal, S. Trebst, S. Wessel, D. Wurtz, M. Troyer, S. Sabhapandit, and S. N. Coppersmith, Phys. Rev. Lett. 92, 097201 (2004).

${ }^{16}$ N. Rathore, Q. L. Yan, and J. J. de Pablo, J. Chem. Phys. 120, 5781 (2004).

${ }^{17}$ E. B. Kim, R. Faller, Q. Yan, N. L. Abbott, and J. J. de Pablo, J. Chem. Phys. 117, 7781 (2002).

${ }^{18}$ M. Doxastakis, Y. L. Chen, and J. J. de Pablo, J. Chem. Phys. 123, 034901 (2005).

${ }^{19}$ L. D. Landau and E. M. Lifshitz, Statistical Physics, 3rd ed. (Pergamon, London, 1980).

${ }^{20}$ J. S. van Duijneveldt and D. Frenkel, J. Chem. Phys. 96, 4655 (1992).

${ }^{21}$ P. R. ten Wolde, M. J. Ruiz-Montero, and D. Frenkel, Phys. Rev. Lett. 75, 2714 (1995).

${ }^{22}$ B. B. Laird and R. L. Davidchack, J. Phys. Chem. B 109, 17802 (2005).

${ }^{23}$ B. B. Laird and R. L. Davidchack, Phys. Rev. Lett. 94, 086102 (2005).

${ }^{24}$ P. G. de Gennes and J. Prost, The Physics of Liquid Crystals (Clarendon, Oxford, 1993).
${ }^{25}$ L. Leibler, Macromolecules 13, 1602 (1980).

${ }^{26}$ P. J. Steinhardt, D. R. Nelson, and M. Ronchetti, Phys. Rev. B 28, 784 (1983).

${ }^{27}$ K. Binder, Phys. Rev. A 25, 1699 (1982).

${ }^{28}$ A. M. Ferrenberg and R. H. Swendsen, Phys. Rev. Lett. 61, 2635 (1988).

${ }^{29}$ C. Borgs and R. Kotecky, Phys. Rev. Lett. 68, 1734 (1992).

${ }^{30}$ M. J. Mandell, J. P. McTaque, and A. Rahman, J. Chem. Phys. 64, 3699 (1976).

${ }^{31}$ C. S. Hsu and A. Rahman, J. Chem. Phys. 71, 4974 (1979).

${ }^{32}$ W. C. Swope and H. C. Andersen, Phys. Rev. B 41, 7042 (1990).

${ }^{33}$ N. B. Wilding and A. D. Bruce, Phys. Rev. Lett. 85, 5138 (2000).

${ }^{34}$ M. B. Sweatman, Phys. Rev. E 72, 016711 (2005).

${ }^{35}$ D. M. Eike, J. F. Brennecke, and E. J. Maginn, J. Chem. Phys. 122, 014115 (2005).

${ }^{36}$ D. Moroni, P. R. ten Wolde, and P. G. Bolhuis, Phys. Rev. Lett. 94, 235703 (2005).

${ }^{37}$ T. Neuhaus and J. S. Hager, J. Stat. Phys. 113, 47 (2003).

${ }^{38}$ L. G. MacDowell, P. Virnau, M. Müller, and K. Binder, J. Chem. Phys. 120, 5293 (2004).

${ }^{39}$ J. E. Mayer and W. W. Wood, J. Chem. Phys. 42, 4268 (1965).

${ }^{40}$ K. Binder and M. H. Kalos, J. Stat. Phys. 22, 363 (1980).

${ }^{41}$ B. A. Berg, U. Hansmann, and T. Neuhaus, Z. Phys. B: Condens. Matter 90, 229 (1993).

${ }^{42}$ J. E. Hunter III and W. P. Reinhardt, J. Chem. Phys. 103, 8627 (1995).

${ }^{43}$ R. L. C. Vink, S. Wolfsheimer, and T. Schilling, J. Chem. Phys. 123, 074901 (2005).

${ }^{44}$ P. R. ten Wolde, M. J. Ruiz-Montero, and D. Frenkel, J. Chem. Phys. 104, 9932 (1996). 